\title{
Water Splitting on Aluminum Porphyrins to form hydrogen and hydrogen peroxide by One-photon of Visible light
}

Fazalurahman Kuttassery, ${ }^{1}$ Shogo Sagawa, ${ }^{1}$ Siby Mathew, ${ }^{1}$ Yu Nabetani, ${ }^{2}$ Akihide Iwase, ${ }^{3}$ Akihiko Kudo, ${ }^{3}$ Hiroshi Tachibana, ${ }^{1}$ and Haruo Inoue ${ }^{1 *}$

${ }^{1}$ Department of Applied Chemistry, Graduate School of Urban Environmental Sciences, Tokyo Metropolitan University, 1-1 Minami-ohsawa, Hachiohji, Tokyo 192-0397 Japan ${ }^{2}$ Deaprtment of Applied Chemistry, Faculty of Engineering, University of Miyazaki, 1-1 Gakuen-kihanadai-nishi, Miyazaki, 889-2192 Japan

${ }^{3}$ Department of Applied Chemistry, Tokyo University of Science, 1-3 Kagurazaka, Shinjyuku-ku, Tokyo 162-0825 Japan

e-mail: inoue-haruo@tmu.ac.jp

\section{Supporting Information}

\section{Fig. S1}

(a)

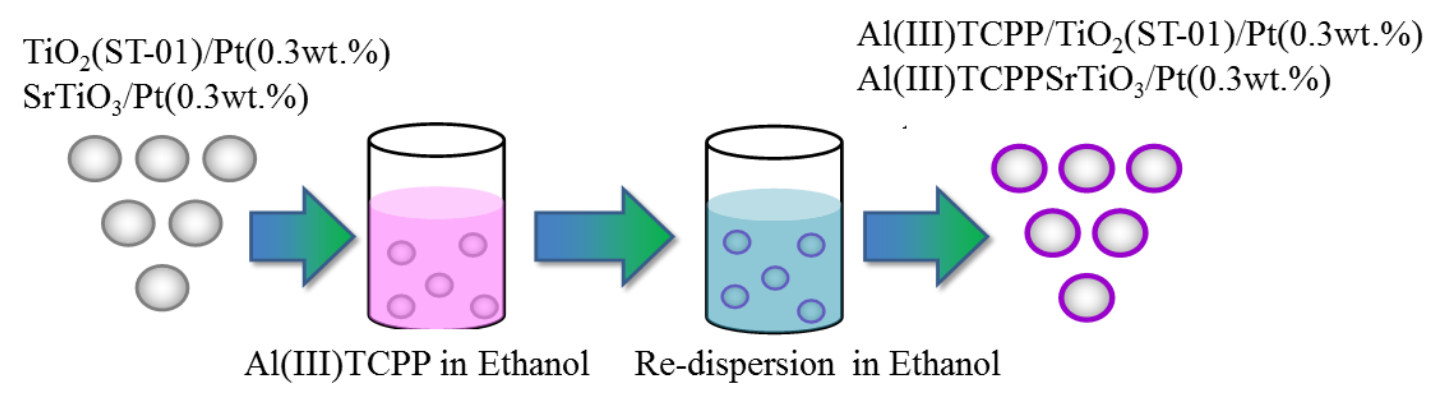

(b)

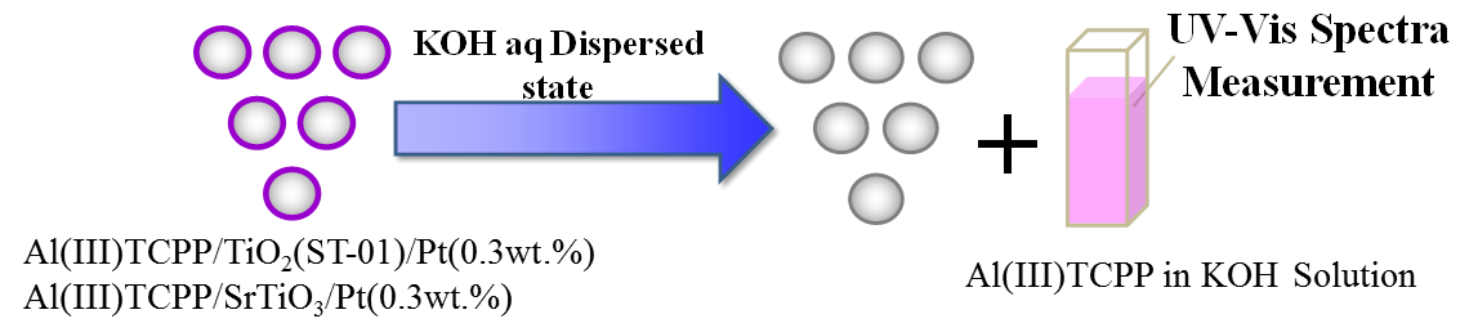

Figure S1. (a) Adsorption procedure of $\mathrm{Al}(\mathrm{III}) \mathrm{TCPP}$ on $\mathrm{TiO}_{2}$ (ST 01)/Pt (0.3 wt.\%) in ethanol solution (b) Desorption of $\mathrm{Al}(\mathrm{III}) \mathrm{TCPP}$ from $\mathrm{Al}(\mathrm{III}) \mathrm{TCPP} / \mathrm{TiO}_{2}(\mathrm{ST} 01) / \mathrm{Pt}(0.3 \mathrm{wt} . \%)$ into sodium potassium hydroxide aqueous solution $(1 \mathrm{M})$ to determine the adsorbed amount of $\mathrm{Al}(\mathrm{III}) \mathrm{TCPP}$. 
Fig. S2

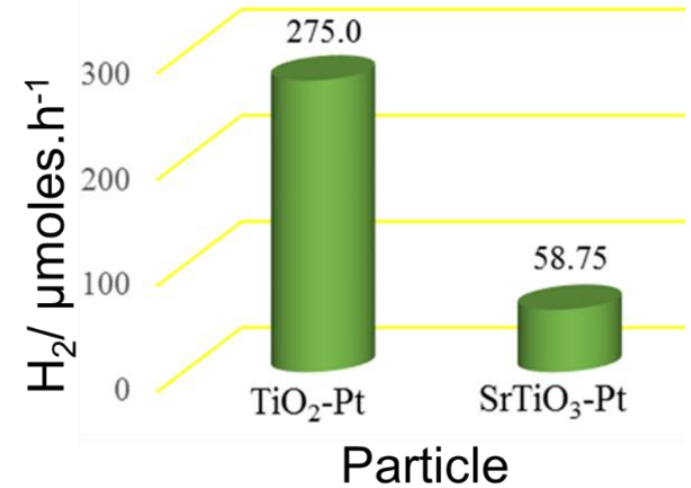

Figure S2. Photochemical hydrogen evolution by light irradiation $(\lambda>290 \mathrm{~nm}$, through IR cut filer) using different semiconductor hybrids $(1 \mathrm{~g} / \mathrm{L})$ in $20 \%$ methanol-water system.

Fig. S3
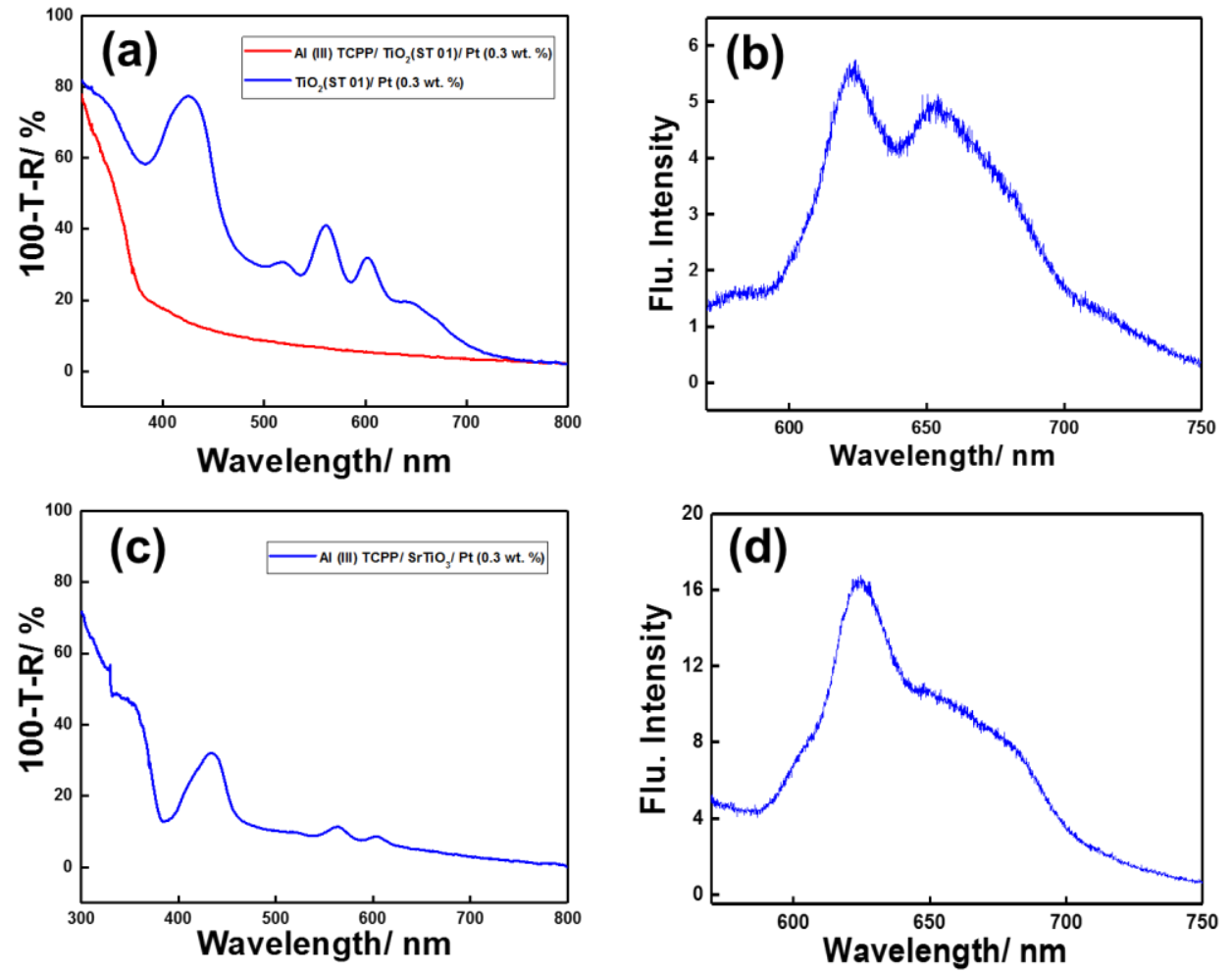

Figure S3. (a) Diffused reflectance spectra of $\mathrm{AlTCPP}_{\mathrm{TiO}}(\mathrm{ST}-01) / \mathrm{Pt}(0.3$ wt. \%) casted film $(4 \mathrm{~g} / \mathrm{L})$ on a cover glass, (b) fluorescence spectra of $\mathrm{AlTCPP}_{\mathrm{TiO}}(\mathrm{ST}-01) / \mathrm{Pt}(0.3 \mathrm{wt} \%)$ as a casted film upon excitation at $430 \mathrm{~nm}$, (c) diffused reflectance spectra of $\operatorname{AlTCPP} / \mathrm{SrTiO}_{3} / \mathrm{Pt}(0.3$ wt. $\%)(4 \mathrm{~g} / \mathrm{L})$ as a casted film on a cover glass, and (d) fluorescence spectra of AlTCPP/SrTiO $3 / \mathrm{Pt}(0.3$ wt. \%) as a casted film upon excitation at $430 \mathrm{~nm}$. 
Fig. S4

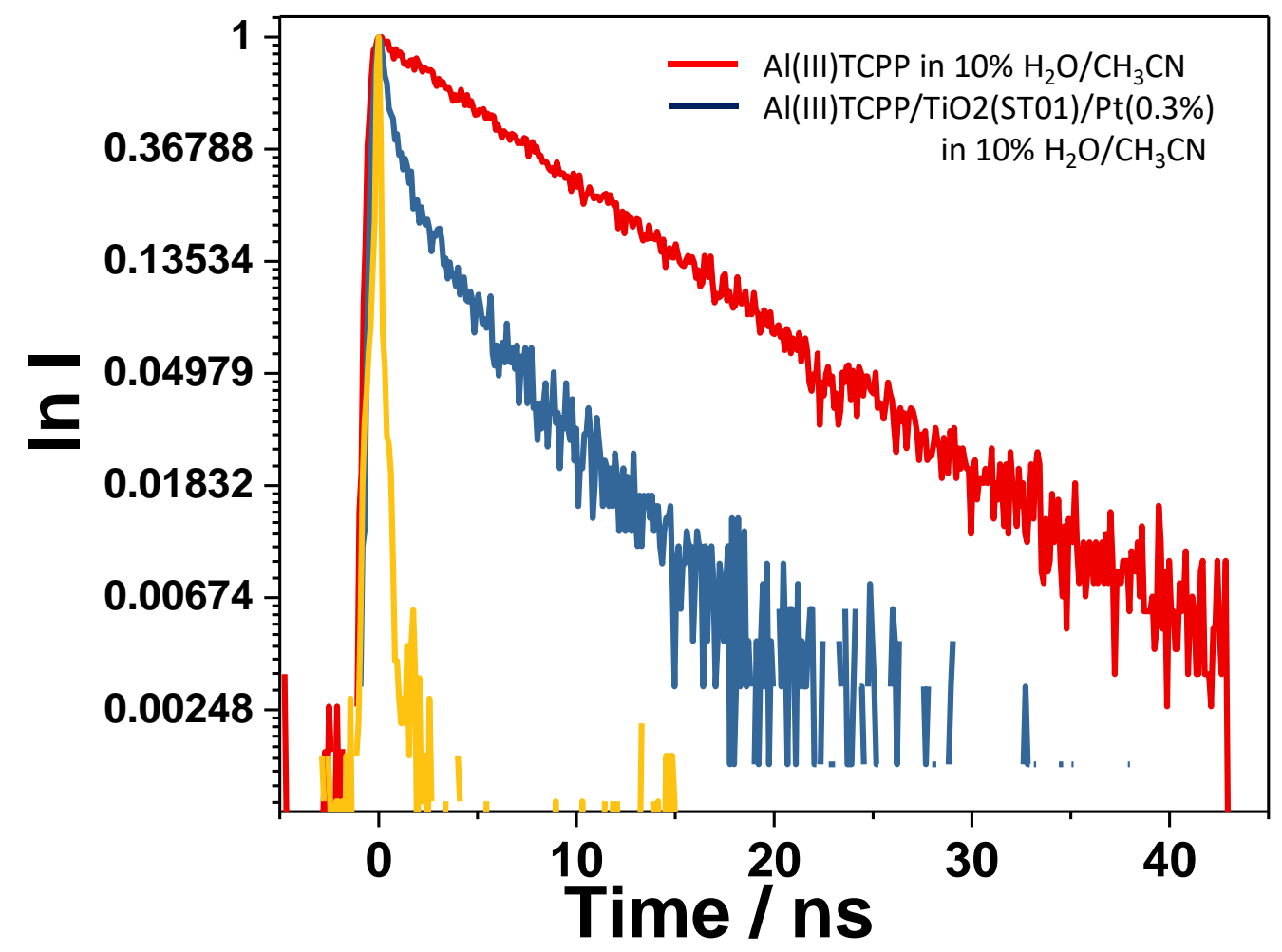

\begin{tabular}{|l|l|l|}
\hline & $\tau_{1}(\mathrm{~ns})$ & $\tau_{2}(\mathrm{~ns})$ \\
\hline $\begin{array}{l}\text { AlTCPP } \\
\text { in } \mathrm{H}_{2} \mathrm{O} / \mathrm{CH}_{3} \mathrm{CN}(1 / 9, \mathrm{v} / \mathrm{v})\end{array}$ & 7.8 & - \\
\hline $\begin{array}{l}\mathrm{AlTCPP} / \mathrm{TiO} 2(\mathrm{ST} 01) / \mathrm{Pt}(0.3 \text { wt. } \%) \\
\text { in } \mathrm{H}_{2} \mathrm{O} / \mathrm{CH}_{3} \mathrm{CN}(1 / 9, \mathrm{v} / \mathrm{v})\end{array}$ & 0.08 & 0.7 \\
\hline
\end{tabular}

Figure S4. Fluorescence decays and lifetimes of AlTCPP in $\mathrm{H}_{2} \mathrm{O} / \mathrm{CH}_{3} \mathrm{CN}(1 / 9, \mathrm{v} / \mathrm{v})$ and $\mathrm{Al}(\mathrm{III}) \mathrm{TCPP} / \mathrm{TiO}_{2}$ (ST 01)/Pt (0.3 wt.\%) dispersion in $\mathrm{H}_{2} \mathrm{O} / \mathrm{CH}_{3} \mathrm{CN}(1 / 9$, v/v). 
Fig. S5

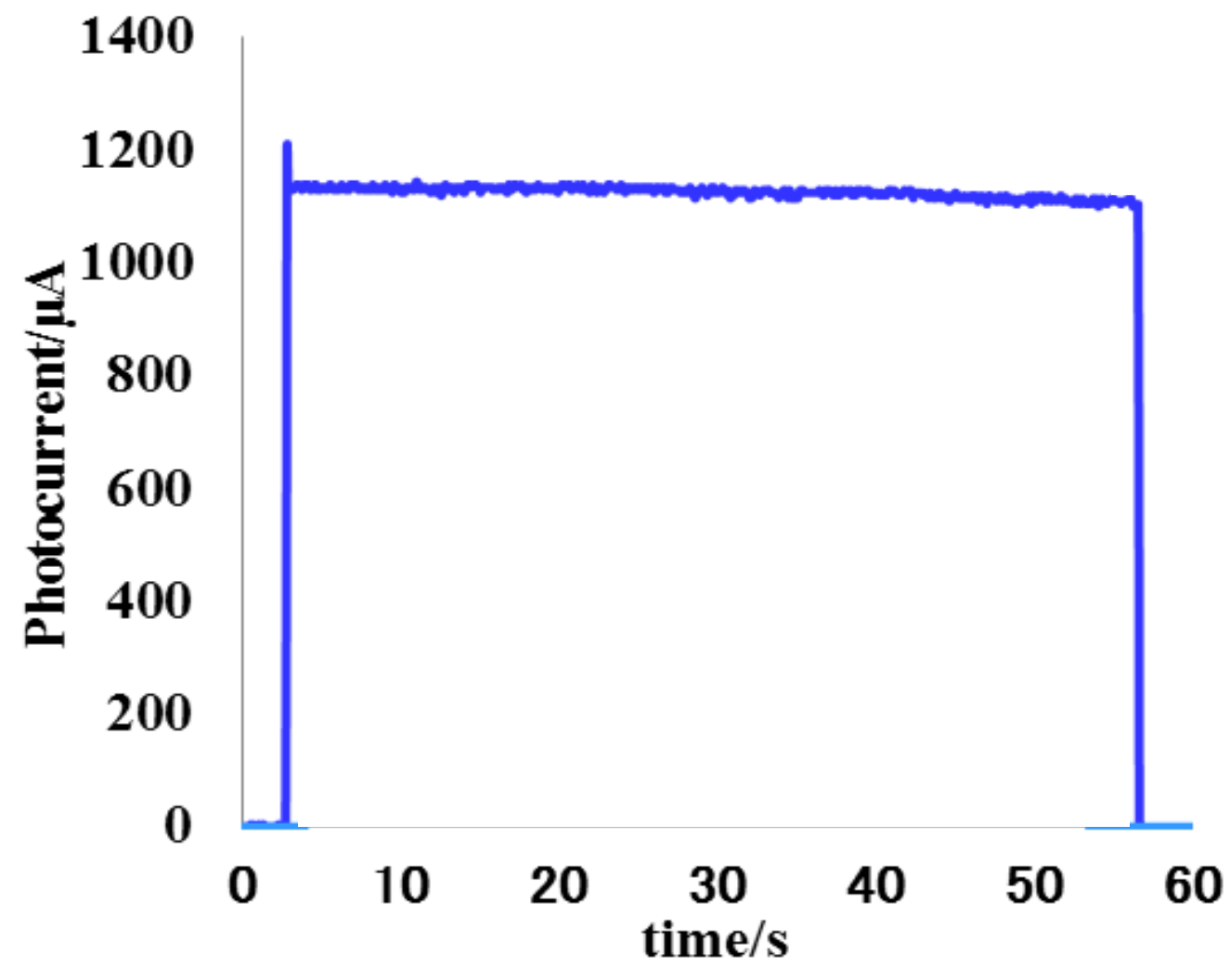

Figure S5. The system, MCSS/photoelectrochemical: Al(III)TCPP was ionically adsorbed on $\mathrm{TiO}_{2}$ nanoparticles (P-25) which were squeezed over fluorinated tin oxide transparent electrode (FTO) and connected with a counter electrode ( $\mathrm{Pt}$ wire) in $\mathrm{H}_{2} \mathrm{O} / \mathrm{CH}_{3} \mathrm{CN}, 1 / 9 \mathrm{v} / \mathrm{v}$ in the presence of tetrapropylammonium iodide (TPAI: $0.2 \mathrm{M}$ ) as the electron mediator. Each electrode, FTO and $\mathrm{Pt}$ was placed in a separated cell connected with salt bridge $\left(\mathrm{agar} / \mathrm{KNO}_{3}(1 \mathrm{M})\right)$. When Soret band of $\operatorname{Al}($ III)TCPP $(\lambda=420 \mathrm{~nm})$ was irradiated with monochromatic visible light $(\lambda=420 \mathrm{~nm}$ from Xe-Lamp through band pass filter for $420 \mathrm{~nm} ; 7.5 \mathrm{mWcm}^{-2}$, area of the light irradiation: $0.984 \mathrm{~cm}^{2}$ ), a high current density up to $\sim 1.2 \mathrm{~mA} \cdot \mathrm{cm}^{-2}$ with absorbed photon to current efficiencies $\sim 50 \%$ (APCE/\%) was observed under the condition. 
Fig. S6

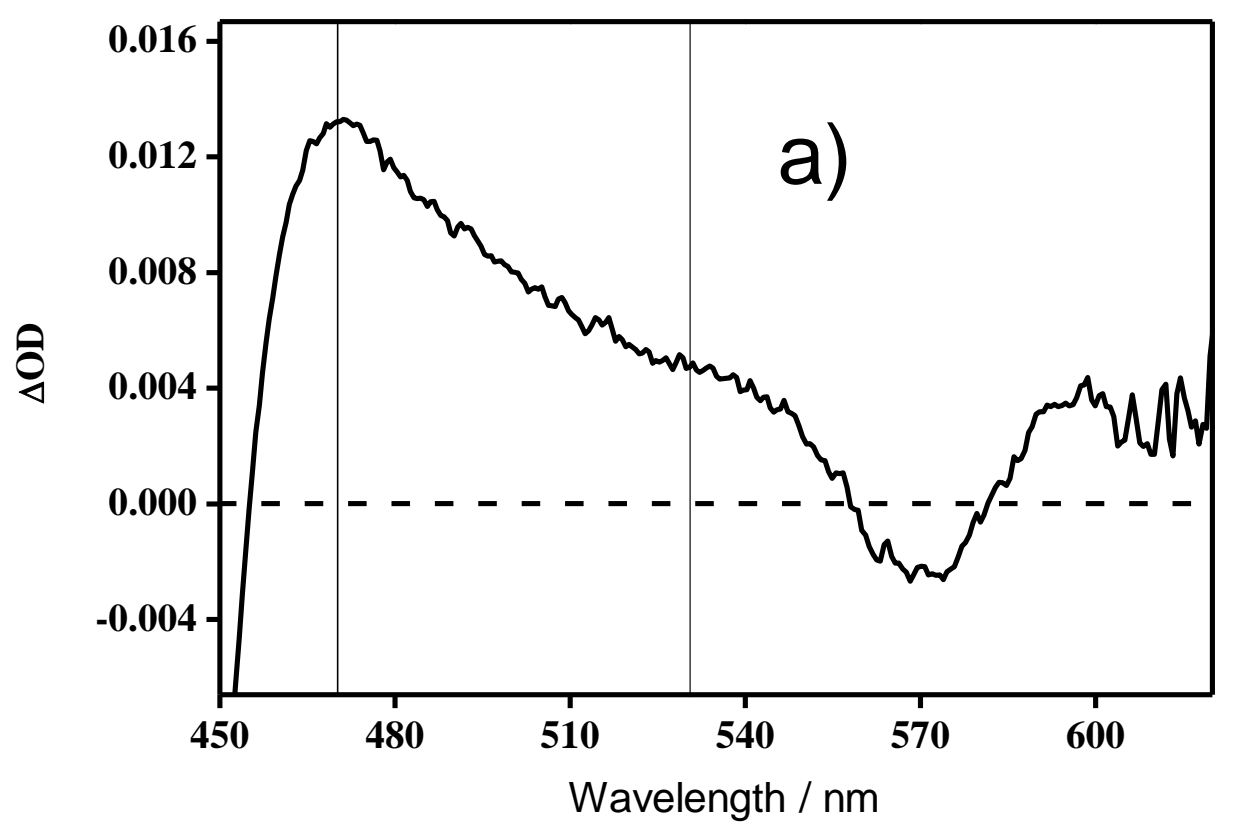

b)

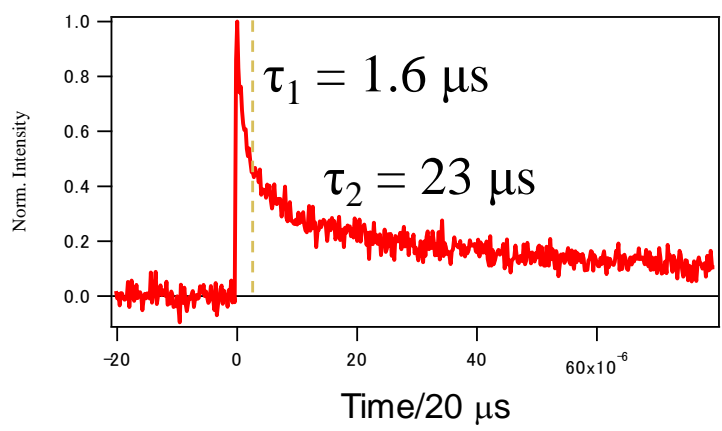

c)

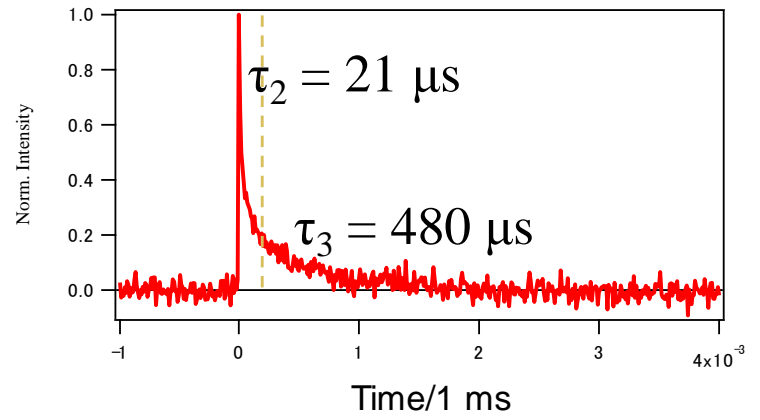

Figure S6 a) Transient absorption spectrum of nano-second laser flash photolysis of MCSS/film ( $\mathrm{AlTCPP} / \mathrm{TiO}_{2}$ ) in $\mathrm{H}_{2} \mathrm{O}$ (phthalate buffer) $/ \mathrm{CH}_{3} \mathrm{CN}(1 / 9, \mathrm{v} / \mathrm{v}$ ) at 0 ns delay time, b) decay profile of the transient absorption at $\lambda=470 \mathrm{~nm}$ within $80 \mu$ s which shows biexponential decays with $\tau_{1}=1.6 \mu \mathrm{s}$ and $\tau_{2}=23 \mu \mathrm{s}$, and c) decay profile within $4 \mathrm{~ms}$ delay which shows biexponential decays with $\tau_{2}=21 \mu \mathrm{s}$ and $\tau_{3}=480 \mu \mathrm{s}$. 
Table S1

Table S1. Estimation of occupied area for AlTCPP adsorbed on various MCSS hybrid particles

\begin{tabular}{|l|l|l|}
\hline Sample & $\begin{array}{l}\text { Adsorbed amount of } \\
\text { AlTCPP } / \mu \mathrm{mol} \cdot \mathrm{g}^{-1}\end{array}$ & $\begin{array}{l}\text { Occupied area of } \\
\text { adsorption/ } \mathrm{nm}^{2} \\
\text { molecule }\end{array}$ \\
\hline AlTCPP/TiO $(\mathrm{ST} 01) / \mathrm{Pt}(0.3 \text { wt. } \%)^{1)}$ & 38.4 & 21.7 \\
\hline $\mathrm{AlTCPP} / \mathrm{SrTiO}_{3} / \mathrm{Pt}(0.3 \text { wt.\% })^{2)}$ & 2.9 & 1.1 \\
\hline
\end{tabular}

1) Specific surface area of $\mathrm{TiO}_{2}(\mathrm{ST} 01)=500 \mathrm{~m}^{2} \cdot \mathrm{g}^{-1}$

2) Specific surface area of $\mathrm{SrTiO}_{3}=2 \mathrm{~m}^{2} \cdot \mathrm{g}^{-1}$

Table S2

Table S2. Stability of hydrogen peroxide in the presence of semiconductor particles: Amounts of free hydrogen peroxide remaining at $30 \mathrm{~min}$ and 150 min after mixing standard $\mathrm{H}_{2} \mathrm{O}_{2}$ aqueous solution $\left(\left[\mathrm{H}_{2} \mathrm{O}_{2}\right]=90 \mu \mathrm{M}\right)$ with various semiconductor particles were estimated by colorimetric analysis using Ti(IV)TPyP.

\begin{tabular}{ccc}
\hline Semiconductor & $\begin{array}{c}\text { Recovered } \\
\mathrm{H}_{2} \mathrm{O}_{2} / \% \\
(30 \mathrm{~min})\end{array}$ & $\begin{array}{c}\text { Recovered } \\
\mathrm{H}_{2} \mathrm{O}_{2} / \% \\
(150 \mathrm{~min})\end{array}$ \\
\hline $\mathrm{TiO}_{2}(\mathrm{ST}-01)$ & 33.7 & 27.8 \\
$\mathrm{TiO}_{2}$ (P-25) & 61.9 & 50.5 \\
$\mathrm{TiO}_{2}$ (Sol-gel) & 69.4 & 58.2 \\
$\mathrm{SrTiO}_{3}$ & 78.9 & 76.7 \\
$\mathrm{TiO}_{2}(\mathrm{ST}-01)-$ & 0.0 & 0.0 \\
$\mathrm{Pt}(0.3$ wt\%) & & \\
$\mathrm{SrTiO}$ (Wako)- & 0.0 & 0.0 \\
$\mathrm{Pt}(0.3$ wt\%) & & \\
\hline
\end{tabular}

